\title{
Underactuated Rehabilitation Robotics for Hand Function
}

\author{
Xuanren Zhu, Bin He \\ Shanghai Key Laboratory of Intelligent Manufacturing and Robotics, \\ School of Mechatronic Engineering and Automation, Shanghai University, China \\ Email: 13817294487@163.com,mehebin@gmail.com
}

\begin{abstract}
At present, the incidence of hand dysfunction caused by diseases such as cerebral palsy or stroke is increasing year by year. For the rehabilitation of hand dysfunction, in addition to surgical treatment, effective rehabilitation exercise is also particularly important. Hand rehabilitation robot is a new rehabilitation instrument developed gradually based on the theory of human rehabilitation medicine, which can effectively treat patients with limb disorders. Therefore, it is necessary to design a safe, stable and portable hand rehabilitation robot. This paper presents a portable and dexterous hand function rehabilitation robot finger joint based on underactuation. This model can reduce the number of drive sources to simplify the mechanical structure, and achieve a separate control function for each finger. In order to verify the correctness of the structure, the kinematic simulation analysis of the skeleton model of the structure is carried out. The results show that the velocity acceleration of the finger changes steadily under the driving of the model, and the bending Angle can reach the specified value. The kinematics model of rehabilitation robot with underactuated hand function designed in this paper is verified to be correct, which can effectively carry out rehabilitation treatment for patients' affected limbs.
\end{abstract}

Keywords-Rehabilitation robot, kinematics simulation, underactuated robotics

\section{INTRODUCTION}

Among patients undergoing surgery due to trauma or hand diseases, hand dysfunction caused by lack of follow-up functional exercise is the most common occurrence [1]. Hand dysfunction will seriously affect patients' daily life, production, labor and other aspects, and will cause great psychological pressure on patients. The rehabilitation process of hand function is very long and requires continuous rehabilitation training of the patient's fingers $[2,3]$. For the former rehabilitation process of hand function, rehabilitation doctors need to continuously perform rehabilitation training of passive bending and stretching of patients' fingers, which greatly increases the workload of rehabilitation doctors and aggravates the strain of medical resources. At the same time, patients in medical institutions for a long period of artificial treatment costs higher, the general family is difficult to bear. Therefore, how to effectively conduct rehabilitation training for patients with hand function, collect rehabilitation data of patients, provide real-time and accurate exercise data for doctors, and intelligently formulate rehabilitation strategies has become an urgent problem to be solved in the field of rehabilitation medicine.
In recent years, robotics has been widely applied in the field of rehabilitation therapy [4-6]. The robotic equipment assists patients' affected limbs to perform repetitive task exercises [7-9], so as to conduct rehabilitation therapy for patients' affected limbs, and the modified treatment method has been proved to be effective in reorganizing the representation of the human motor cortex [10,11]. For patients with hand dysfunction, hand function rehabilitation robot is gradually used for auxiliary training to achieve the rehabilitation effect of the hand. Compared with traditional rehabilitation treatment methods, manual rehabilitation robot can effectively relieve the strain of medical resources in hospitals and reduce the cost of rehabilitation training for patients with hand function. At the same time, it can obtain the data signal of the patient's hand stress and bend, so as to realize intelligent rehabilitation training. To develop a user system of the rehabilitation robot, it can monitor the patient's recovery status in real-time on the computer. It can also make it more convenient for rehabilitators or patients.

The research and development of hand-functional rehabilitation robots combines rehabilitation medicine $[12,13]$, robotics [14], computer technology [15,16], structural mechanics [17], bionics [18] and other fields. At present, hand functional rehabilitation robots can be divided into two categories according to different materials: rigid hand functional rehabilitation robots [19-21] and flexible hand functional rehabilitation robots [22-24]. The research and development of hand functional rehabilitation robot is also a challenging and practical field, for which many researchers have done a lot of research work. Yongkang Jiang et al. [25] designed a hand functional rehabilitation robot with fishbone biomimetic structure based on PneuNets bend actuators [26], and realized different bending shapes to assist finger bending during soft brake expansion. Arata et al. [27] used a three-layer sliding spring mechanism to develop a set of flexible hand-functional rehabilitation robot, which controlled the spring sheet to flex in the outer structure through a single motor, so as to control the bending of the hand-functional rehabilitation robot. Haghshenas et al. [28] developed a flexible hand functional rehabilitation robot based on a hybrid brake system, using a flexible bellows-type soft brake and a semi-rigid structure [29].

At present, there are still many technical difficulties to overcome, such as complex mechanical structure; It is inconvenient for patients to wear; Each finger cannot perform a separate rehabilitation movement; The device adopts rigid 
devices and structures, and its safety needs to be improved, but it can provide enough driving force to help patients with rehabilitation training. The flexible hand rehabilitation robot has the advantages of light structure, high safety, and low cost [30]. However, its target diseases of hand dysfunction are limited, and most of them are targeted at stroke patients. Therefore, it is not suitable for the rehabilitation of some patients with stiff fingers due to its small bending force.

This work presents a finger joint model of a portable rehabilitation robot with dexterity and stable strength. Reasonable underactuated mechanism [31] can effectively reduce the complexity of the structure, reduce the weight of the structure, and the motion is flexible. In this paper, the kinematics model of the single finger joint of the hand functional rehabilitation robot is designed. By constructing the skeleton model of the underactuated hand rehabilitation robot, the kinematics and dynamics of the robot are simulated and analyzed.

The other contents of this paper are as follows: In Section 2 , the motion diagram of the rehabilitation robot with hand function is constructed and its kinematic principle is analyzed; in Section 3, the virtual prototype simulation model of the rehabilitation robotics for hand function is established and its kinematic simulation is analyzed; in Section 4, the conclusion is drawn.

\section{ESTABLISHMENT OF KINEMATICS MODEL OF HAND- FUNCTIONAL REHABILITATION ROBOT}

For the design and implementation of the rehabilitation robot with hand function, we must first consider several design principles: It can support rehabilitation training of 2-5 metacarpal and phalangeal joints, and realize passive bending training of fingers and independent control of joints. It is simple to wear and can be sized to fit or adjust the palm of a normal adult.

Based on the above research foundation, it is necessary to design a hand functional rehabilitation robot based on the miniaturization of underactuated mechanism, multi-function, multi-freedom design, sensor system integration, and other comprehensive aspects. Before designing the mechanical structure of hand functional rehabilitation, it is necessary to determine the degree of freedom of the mechanism. For the hand function rehabilitation robot, it needs to be worn on the human hand, so it needs to meet the freedom degree of each finger activity. However, according to rehabilitation needs, the flexion of metacarpophalangeal joint and middle knuckle should be rehabilitated, without considering the adduction and abduction of metacarpophalangeal joint of 2-5 knuckles. Therefore, the free degree of the 2-5 finger single-finger actuator of the manual functional rehabilitation robot is positioned at 2 degrees of freedom. However, due to the small space of the hand, the underactuated structure is considered to reduce the number of actuators.

The kinematics model of the rehabilitation robot is established, the coordinate system of the hand-functional rehabilitation robot is determined, and the components of the hand-functional rehabilitation robot are simplified. The components and motions of proximal joint actuators and distal joint actuators are named. Since the principle of kinematics analysis of five fingers is the same, this paper takes the index finger actuator as an example to carry out kinematics analysis. The flexion and extension of the index finger joints around the Z-axis are considered only, and the adduction and abduction of the index finger joints around the Y-axis are not considered. The kinematics model of the forefinger functional rehabilitation robot is shown in Fig 1. Points A, B and C are metacarpophalangeal joint, proximal interphalangeal joint and distal interphalangeal joint respectively. $\mathrm{AB}$ represents the proximal knuckles, $\mathrm{BC}$ represents the middle knuckles, $\theta_{1}$ is the Angle at which the proximal knuckles bend, and $\theta_{2}$ is the Angle at which the middle knuckles rotate. $l_{2}$ bar is used as the power input in the driving mechanism of the proximal knuckle, and the rotation of $l_{2}$ bar drives the movement of a-bar, thus driving the proximal knuckle to bend. The movement of $l_{2}$ bar can be converted into the form of sliding guide bar, which is driven by the electric push bar in the designed sliding guide rail. This drives the proximal knuckle to bend at the corresponding Angle. The mechanism has a fixed trajectory and good controllability and can control the bending Angle of the proximal knuckle by controlling the expansion and expansion of the electric push rod. The connecting rod in the mid-end knucklehead actuator is respectively represented by a, b, c, and $\mathrm{d}$. The motion of the a-bar is the input, while the other three bars are not fixed, so the actuator of the mid-end joint is underdriven. The second section USES underactuated structure, which reduces the number of driving elements and has good adaptability to the movement of the middle knuckle.

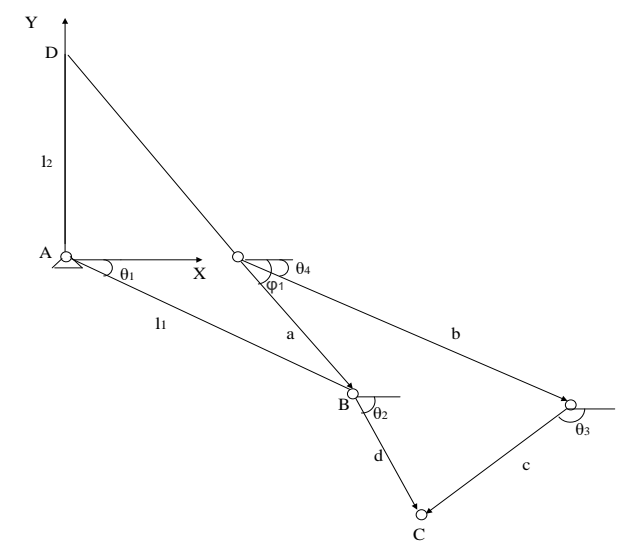

Fig. 1. Kinematics model diagram of hand functional rehabilitation robot.

In Figure 1, point B of the proximal interphalangeal joint can be uniquely determined by the motion of $\operatorname{rod} 1_{1}$. When $\theta_{1}$ is changed, the coordinates of point $\mathrm{B}$ can be expressed as:

$$
\begin{aligned}
& \mathrm{X}_{\mathrm{B}}=\mathrm{l}_{1} * \cos \theta_{1} \\
& \mathrm{Y}_{\mathrm{B}}=\mathrm{l}_{1} * \sin \theta_{1}
\end{aligned}
$$

The motion of the proximal knuckle brake is relatively simple, and the position, speed and acceleration of point B are related to the input speed of the electric push rod. It can be seen from the model of the hand functional rehabilitation robot in chapter 2 that the connection of the electric putter is point $\mathrm{D}$. Set the driving speed of the electric push rod as $v_{1}$, and the angular velocity of the proximal knuckle motion can be pushed as follows:

$$
\omega_{1}=\mathrm{v}_{1} / \mathrm{l}_{2}
$$


The middle knuckle actuator is a vector quadrilateral composed of $\{a, b, c, d\}$. From the vector relation, it can be obtained as follows:

$$
\begin{aligned}
& a \cos \varphi_{1}+d \cos \theta_{2}-c \cos \theta_{3}-b \cos \theta_{4}=0 \\
& a \sin \varphi_{1}+d \sin \theta_{2}-c \sin \theta_{3}-b \sin \theta_{4}=0
\end{aligned}
$$

Where $\varphi_{1}$ is the fixed value controlled by the structure movement of the proximal knuckle. $\theta_{4}$ is the input to the underdriven structure; The values of $\theta_{2}$ and $\theta_{3}$ are unknown, and you need to find the corresponding expressions for them by using the corresponding equations. By calculating the first derivative and the second derivative of the formula, the related information of the structural velocity can be obtained

$$
\left[\begin{array}{cc}
-d \sin \theta_{2} & c \sin \theta_{3} \\
d \cos \theta_{2} & -c \cos \theta_{3}
\end{array}\right] *\left[\begin{array}{c}
\dot{\theta}_{2} \\
\dot{\theta}_{3}
\end{array}\right]=\dot{\theta}_{4}\left[\begin{array}{c}
-b \sin \theta_{4} \\
b \cos \theta_{4}
\end{array}\right]
$$

\section{KINEMATIC SIMULATION ANALYSIS OF HAND- FUNCTIONAL REHABILITATION ROBOT}

Overall structural design mainly have two bottom-up and top-down design principle, the structure of the top-down design principle is through the design goal to establish virtual prototype model of a product, virtual prototype model function structure analysis, verify its performance index, based on the analysis of constantly optimize the structure, top-down design principle can effectively improve the quality of the design of the product, greatly shorten the design cycle of the product. Therefore, after designing the mechanical structure of the functional rehabilitation robot, it is necessary to carry out the simulation analysis of the dynamics and kinematics of the mechanical structure. Analyze information on the position, velocity, acceleration, and force of the recovery manipulator. After analyzing the structure to meet the performance indexes of the manual functional rehabilitation robot, the mechanical parts of the manual functional rehabilitation robot can be processed and assembled. If the performance indicators of rehabilitation training cannot be reached, the mechanical structure shall be improved until the simulation results meet the indicators of rehabilitation training.

Based on the design objective and basic function model of the hand-functional rehabilitation robot, the corresponding skeleton model is constructed in ADAMS, and then the corresponding constraints and driving conditions are set to carry out kinematic simulation analysis on the simulation model. For the hand functional rehabilitation robot, the skeleton model is composed of different spatial components, which ignores the specific geometric shape and various dimensional details of the 3D model and only needs to define the constraint relationship between its spatial components and key geometric dimensions, which greatly improves the efficiency of simulation analysis and gets relatively accurate calculation results.

The structure design optimization process of the skeleton model based on the manual functional rehabilitation robot is shown in Fig 2. First of all, based on domestic and foreign product research, technical research, and other work to determine the product design objectives; According to the design objective, the two-dimensional motion diagram and kinematic model of the product are constructed, and the kinematic principle is analyzed. The skeleton model is built based on virtual prototyping technology, and the kinematics simulation analysis is carried out to verify whether the design meets the requirements. If it meets the requirements, the detailed design of the 3D model is carried out; if not, the kinematics model of the hand-functional rehabilitation robot is redesigned.

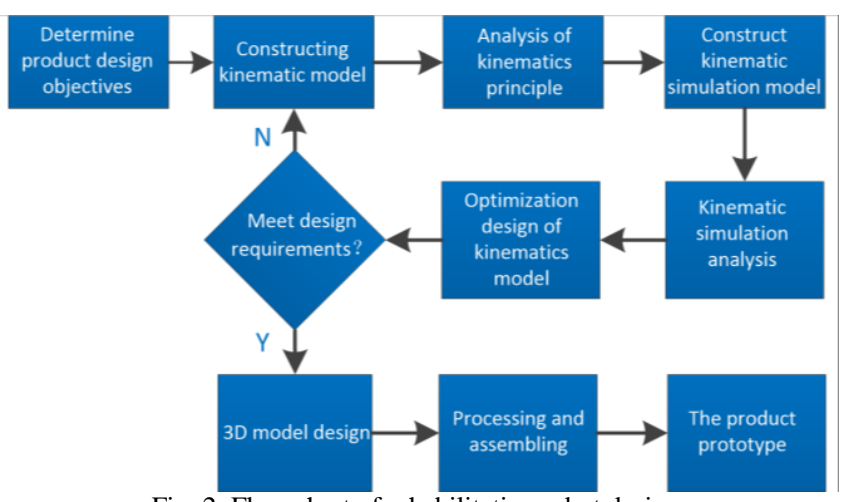

Fig. 2. Flow chart of rehabilitation robot design.

The dynamic analysis results of the skeleton model based on the hand-functional rehabilitation robot are shown in Fig 3. The motion trajectory of finger joints can be determined by the joint coordinates. The $\mathrm{X}$-axis and $\mathrm{Y}$-axis coordinates of the proximal knuckle and middle knuckle joints were measured. Sh1i_x is the $\mathrm{X}$-axis coordinate of the proximal knuckle in the coordinate system with the origin of the index finger joint, shi1_y is the Y-axis coordinate of the proximal knuckle in the coordinate system, shi2_x is the $\mathrm{X}$-axis coordinate of the middle knuckle in the coordinate system, and Shi2_y is the Y-axis coordinate of the middle knuckle in the coordinate system.

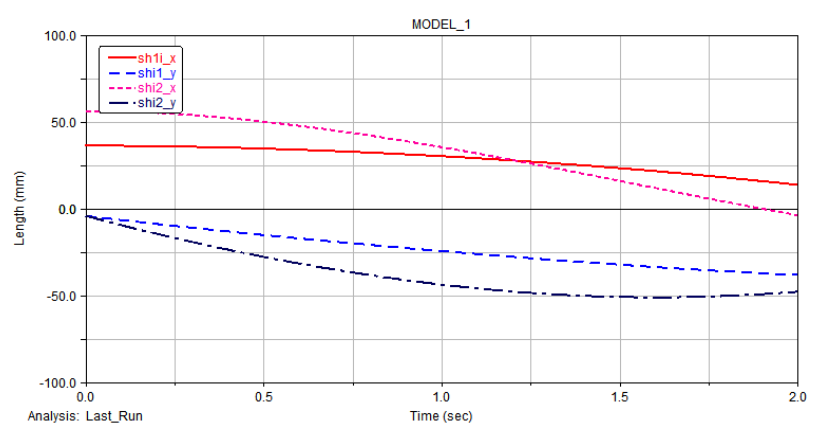

Fig. 3. Coordinate chart of motion of each knuckler.

The $\mathrm{X}$-axis coordinate of the two knuckles is taken as the $\mathrm{X}$-axis parameter of the curve, and the $\mathrm{Y}$-axis coordinate of the two knuckles is taken as the $\mathrm{Y}$-axis parameter of the curve. Under the action of the finger-hand functional rehabilitation robot, the coordinate trajectories of each joint can be plotted, as shown in Fig 4. 


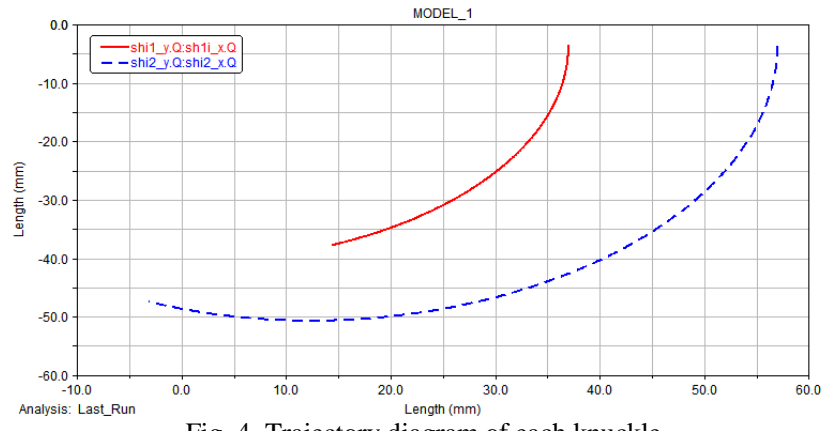

Fig. 4. Trajectory diagram of each knuckle.

The trajectory curve of finger joints during movement can be measured and plotted, and the movement of fingers under the action of the hand function rehabilitation robot can be roughly seen. By measuring the rotation angle of each joint in the process of moving, the relationship between the rotation angle of the finger in the process of motion and the time and the stretching amount of the electric push rod can be seen more intuitively, which is of great help to the motion control in the later stage. (a) is the relation curve of the bending angle of proximal knuckle over time. (b) is the relation curve of the bending Angle of the middle knuckle over time.
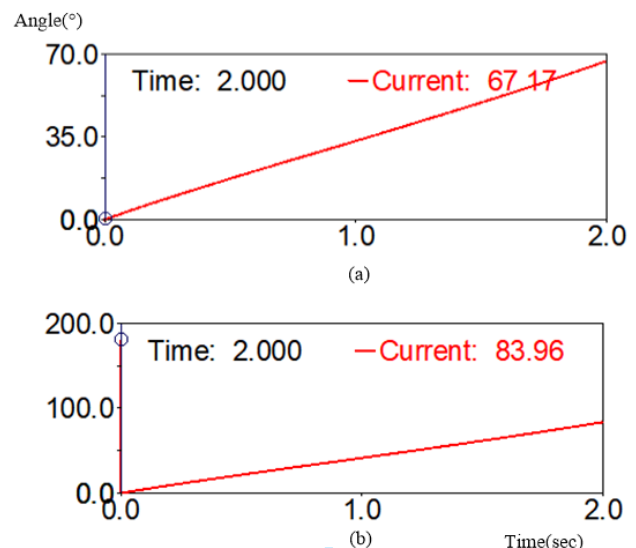

Fig. 5. Curve of proximal and middle knuckle bending angles.

When the movement time is set at $2 \mathrm{~s}$, that is, the push-rod can be extended by $20 \mathrm{~mm}$. The maximum bending angle of the proximal knuckle was $67.17^{\circ}$, and the maximum bending Angle of the middle knuckle was $83.96^{\circ}$. It can reach the performance index set by the manual rehabilitation robot. In addition, it is necessary to measure the angular velocity and angular acceleration of the finger in the process of motion. The angular velocity, angular acceleration, and angular acceleration of the node between the proximal knuckle and the distal knuckle are measured.

As shown in Fig 6 below, shi1_jiaos is the angular velocity of the proximal knuckle rotation, shi2_jiaos is the angular velocity of the middle knuckle rotation, shi1_jiaoa is the angular velocity of the proximal knuckle rotation, and shi2_jiaoa is the angular acceleration of the middle knuckle rotation. The structure can ensure the safety of patients in the process of rehabilitation.

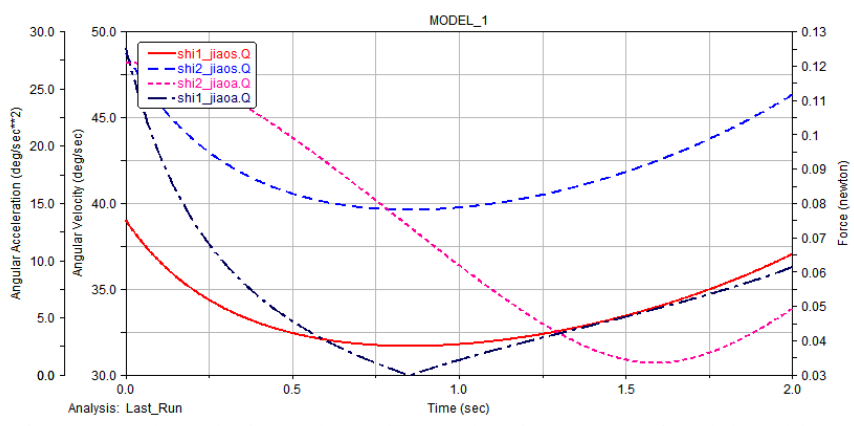

Fig. 6. Angular velocity and angular acceleration curves of each knuckle.

\section{CONCLUSION}

This work presents a finger joint model of a portable rehabilitation robot with dexterity and stable strength. Through the design and kinematic simulation analysis of finger joints of the rehabilitation robot with hand function, and the comparison of design objectives and simulation results, it is concluded that the designed structure can effectively rehabilitate patients with hand function disorder. The device adopts underactuated mechanism driving mode, which improves the portability and flexibility of the device and reduces the complexity of the structure. Through kinematic and dynamic simulation analysis, the results show that the maximum bending Angle of the proximal knuckle of the mechanism is 67.17 degrees, and the maximum bending Angle of the middle knuckle is 83.96 degrees. It can reach the performance index set by the manual rehabilitation robot, and the angular velocity and angular acceleration of the two joints change gently in the process of motion, which verifies the correctness of the mechanism.

In the hands of traditional rigid rehabilitation machinery, a fully driven structure is usually adopted [32], that is, a driving unit is added to each curved joint of a single finger, which greatly increases the complexity of the structure. At the same time, due to the addition of driving units in each finger joint, the matching requirements of the finger bending joint position are extremely high, which cannot be well adapted to rehabilitation training of different human hand size, and the adaptability is not high. In this paper, the underactuated structure design is carried out for the hand functional rehabilitation robot to reduce the number of power sources of the mechanism, so that the structure of the hand functional rehabilitation robot can be simplified and move flexibly. In addition, the matching requirements for bent joints are not high, and it can be adapted to wear with different finger sizes.

\section{ACKNOWLEDGEMENTS}

The work was supported by Shanghai Science and Technology Committee Project (Grant No.19441908602). Thanks for the help from the team of Prof. Lei Xu, Huashan Hospital, Fudan University.

\section{REFERENCES}

[1] M. Li et al., "A soft robotic glove for hand rehabilitation training controlled by movements of the healthy hand," 2020 17th International Conference on Ubiquitous Robots (UR), Kyoto, Japan, 2020, pp. 6267.

[2] P. Jacqueline A, H. Alice, G. Whitney, W. Steven L, and B. Michael $\mathrm{R}$, "Modulatory effects of motor state during paired associative 
stimulation on motor cortex excitability and motor skill learning." Front. Hum. Neurosci., vol. 13, 2019.

[3] S. W. James , and W. D. Byblow, "Disinhibition in the human motor cortex is enhanced by synchronous upper limb movements," The $\mathrm{J}$ Physiol., vol. 543, no. 1, pp. 307-316, 2004.

[4] J. Lee, S. Kim, W. Park and J. Bae, "Design of a wearable hand exoskeleton system for evaluation of hand functions," 2017 14th International Conference on Ubiquitous Robots and Ambient Intelligence (URAI), Jeju, 2017, pp. 578-579.

[5] A. Nontapha and W. San-Um, "A versatile hand grip design for concise arm and hand rehabilitation robot for stroke patients," 2017 Third International Conference on Biosignals, Images and Instrumentation (ICBSII), Chennai, 2017, pp. 1-4.

[6] J. Ma, R. Mo, M. Chen, L. Cheng and H. Qi, "Mirror-Training of a Cable- Driven Hand Rehabilitation Robot Based on Surface Electromyography (sEMG)," 2019 Tenth International Conference on Intelligent Control and Information Processing (ICICIP), Marrakesh, Morocco, 2019, pp. 309-315.

[7] N. G. Kutner, R. Zhang, A. J. Butler, S. L. Wolf, and J. L.Alberts, "Quality-of-life change associated with robotic-assisted therapy to improve hand motor function in patients with subacute stroke: a randomized clinical trial," Phys. Ther., vol. 90, no. 4, pp. 493-504, 2010.

[8] Y. Ren, Y. -N. Wu, C. -Y. Yang, T. Xu, R. L. Harvey and L. -Q. Zhang, "Developing a Wearable Ankle Rehabilitation Robotic Device for inBed Acute Stroke Rehabilitation," in IEEE Transactions on Neural Systems and Rehabilitation Engineering, vol. 25, no. 6, pp. 589-596, 2017.

[9] A. Borboni, J. H. Villafane, C. Mullè, K. Valdes, R. Faglia, G. Taveggia, and S. Negrini, "Robot-assisted rehabilitation of hand paralysis after stroke reduces wrist edema and pain: a prospective clinical trial," J. Manipulative Physiol. Ther., vol. 40, no. 1, 2016.

[10] C. D. Takahashi, L. Der-yeghiaian, V. Le, R. R. Motiwala, and S. C. Cramer, "Robot-based hand motor therapy after stroke," Brain, vol. 131, no. 2, pp. 425-437, 2008.

[11] S. Umchid and P. Taraphongphan, "Design and development of a smart continuous passive motion device for knee rehabilitation," 2016 9th Biomedical Engineering International Conference (BMEiCON), Laung Prabang, 2016, pp. 1-5.

[12] Slipman, and W. Curtis, "Rehabilitation medicine: principles and practice," JAMA., vol. 271, no.4, pp.244, 1994.

[13] M. Gandolfi, C. Geroin, A. Waldner, I. Maddalena, C. Tomelleri, "Feasibility and safety of early lower limb robot-assisted training in sub-acute stroke patients: a pilot study," Eur. J. Phy3. Rehabil. Med., vol. 53, no. 6, 2017.

[14] J. Oliver, R. Toledo and E. Valderrama, "A learning approach based on robotics in Computer Science and Computer Engineering," IEEE EDUCON 2010 Conference, Madrid, 2010, pp. 1343-1347.

[15] H. Wang and J. Gu, "Application of Deep Learning in Humancomputer Interaction Design of Rehabilitation Equipment," 2019 2nd International Conference on Information Systems and Computer Aided Education (ICISCAE), Dalian, China, 2019, pp. 99-102.

[16] A. Benzerrouk, L. Adouane, L. Lequievre and P. Martinet, "Navigation of multi-robot formation in unstructured environment using dynamical virtual structures," 2010 IEEE/RSJ International Conference on Intelligent Robots and Systems, Taipei, 2010, pp. 5589-5594.

[17] R. Michael, T. Bertram, and M. Iwasaki, "Modeling, observation, and control of hysteresis torsion in elastic robot joints," Mechatronics, vol. 24, no. 5, pp. 407-415, 2014.
[18] J. He, and F. Gao, "Type synthesis for bionic quadruped walking robots," J. Bionic. Eng., vol. 12, no. 004, pp. 527-538, 2015.

[19] X. Li, Y. H. Liu, and H. Y. Yu, "Iterative learning impedance control for rehabilitation robots driven by series elastic actuators." Automatica, vol. 90, pp.1-7, 2018.

[20] K. Shi, A. Song, Y. Li and C. Wu, "Cable-driven Wearable Upper Limb Rehabilitation Robot," 2018 IEEE International Conference on Robotics and Biomimetics (ROBIO), Kuala Lumpur, Malaysia, 2018, pp. 2434-2438.

[21] P. Wattanasiri, P. Tangpornprasert and C. Virulsri, "Design of MultiGrip Patterns Prosthetic Hand With Single Actuator," in IEEE Transactions on Neural Systems and Rehabilitation Engineering, vol. 26, no. 6, pp. 1188-1198, June 2018.

[22] B. Wang, A. McDaid, K. C. Aw and M. Biglari-Abhari, "Design and development of a skinny bidirectional soft glove for post-stroke hand rehabilitation," 2017 Intelligent Systems Conference (IntelliSys), London, 2017, pp. 979-987.

[23] J. Zhang, H. Wang, J. Tang, H. Guo and J. Hong, "Modeling and design of a soft pneumatic finger for hand rehabilitation," 2015 IEEE International Conference on Information and Automation, Lijiang, 2015, pp. 2460-2465.

[24] M. Ariyanto, J. D. Setiawan, R. Ismail, I. Haryanto, and D. R. Saksono, "Design and characterization of low-cost soft pneumatic bending actuator for hand rehabilitation," 2018 5th International Conference on Information Technology, Computer, and Electrical Engineering, 2018.

[25] Y. Jiang et al., "Fishbone-inspired soft robotic glove for hand rehabilitation with multi-degrees-of-freedom," 2018 IEEE International Conference on Soft Robotics (RoboSoft), Livorno, 2018, pp. 394-399.

[26] H. Lipson, "Challenges and opportunities for design, simulation, and fabrication of soft robots," Soft Robotics, vol. 1, pp. 21-27, 2014.

[27] J. Arata, K. Ohmoto, R. Gassert, O. Lambercy, H. Fujimoto and I. Wada, "A new hand exoskeleton device for rehabilitation using a threelayered sliding spring mechanism," 2013 IEEE International Conference on Robotics and Automation, Karlsruhe, 2013, pp. 39023907.

[28] M. Haghshenas-Jaryani, W. Carrigan, C. Nothnagle and M. B. J. Wijesundara, "Sensorized soft robotic glove for continuous passive motion therapy," 2016 6th IEEE International Conference on Biomedical Robotics and Biomechatronics (BioRob), Singapore, 2016, pp. 815-820.

[29] P. Hahn, H. Krimmer, A. Hradetzky, and U. Lanz, "Quantitative analysis of the linkage between the interphalangeal joints of the index finger. An in vivo study," J. Hand Surg. Br., vol. 20, no. 5, pp. 696699, 1995.

[30] Z. Xue, Q. Wu and F. Gao, "Design and Modeling of Omni-directional Bending Pneumatic Flexible Arm," 2018 3rd International Conference on Advanced Robotics and Mechatronics (ICARM), Singapore, 2018, pp. 835-839.

[31] K. Gao, F. Yang, M. Zhou, Q. Pan and P. N. Suganthan, "Flexible JobShop Rescheduling for New Job Insertion by Using Discrete Jaya Algorithm," in IEEE Transactions on Cybernetics, vol. 49, no. 5, pp. 1944-1955, May 2019.

[32] J. T. LI, S. Wang, J. Wang, R. Y. Zheng, Y. R. Zhang, and Z. Y. Chen, "Development of a hand exoskeleton system for index finger rehabilitation,” J. Mech. Eng., vol. 02, pp. 223-233, 2012. 